Network Working Group

Request for Comments: 2316

S. Bellovin

Category: Informational

\title{
Report of the IAB Security Architecture Workshop
}

1. Status of this Memo

This memo provides information for the Internet community. It does not specify an Internet standard of any kind. Distribution of this memo is unlimited.

2. Copyright Notice

Copyright (C) The Internet Society (1998). All Rights Reserved.

3. Abstract

On 3-5 March 1997, the IAB held a security architecture workshop at Bell Labs in Murray Hill, NJ. We identified the core security components of the architecture, and specified several documents that need to be written. Most importantly, we agreed that security was not optional, and that it needed to be designed in from the beginning .

\subsection{Specification Language}

The key words "MUST", "MUST NOT", "REQUIRED", "SHALL", "SHALL NOT", "SHOULD", "SHOULD NOT", "RECOMMENDED", "MAY", and "OPTIONAL" in this document are to be interpreted as described in RFC 2119.

4. Motivations

On 3-5 March 1997, the IAB held a security architecture workshop at Bell Labs in Murray Hill, NJ. The ultimate goal was to design a security architecture for the Internet. More concretely, we wished to understand what security tools and protocols exist or are being developed, where each is useful, and where we are missing adequate security tools. Furthermore, we wanted to provide useful guidance to protocol designers. That is, if we wish to eliminate the phrase "security issues are not discussed in this memo" from future RFCs, we must provide guidance on acceptable analyses. 
There were twenty-four attendees (their names are listed in Appendix A). Perhaps not surprisingly for such a group, the overwhelming majority used some form of cryptography when connecting back to their home site from the meeting room. But the situation on the rest of the Internet is not nearly as good; few people use encryption, even when they should.

The problem is that the rate of attacks is increasing. Apart from the usual few elite hackers -- the ones who find the new holes -there are many canned exploit scripts around. ("Click here to attack this system.") Furthermore, the attackers have gotten smarter; rather than going after random university machines, more and more are targeting the Internet infrastructure, such as routers, high-level name servers, and the like.

The problem is compounded by organizational laziness. Users and system administrators want "magic security" -- they want whatever they do to be secure, regardless of whether or not it is, or even can be.

5. General Philosophy

We concluded that in general, end-to-end security is better. Thus, one should use something like PGP or S/MIME for email, rather than relying on an IPsec layer. In general, relying on the security of the infrastructure is a bad idea; it, too, is under attack. Even firewall-protected intranets can be subverted. At best, the infrastructure should provide availability; it is the responsibility of individual protocols not to make unreasonable demands on the infrastructure during an attack.

6. IETF Structure

Our security problem is compounded by the IETF's inherent structure (or, in some cases, the lack thereof). By intent, we are a volunteer organization. Who should do the security work? The other protocol designers? Often, they have neither the time nor the interest nor the training to do it. Security area members? What if they are not interested in some subject area, or lack the time themselves? We cannot order them to serve.

To the extent that the IETF does have management, it is embodied in the working group charters. These are in essence contracts between the IESG and a working group, spelling out what is to be done and on what schedule. Can the IESG unilaterally impose new requirements on existing working groups? What if security cannot be added on without substantial changes to the fundamental structure of a protocol that has been reworked over several years? 
Finally, there is a perception problem: that IPsec will somehow solve the security problem. It won't; indeed, it can't. IPsec provides excellent protection of packets in transit. But it's hard to deploy on individual hosts, does not protect objects that may be retransmitted (i.e., email messages), does not address authorization issues, cannot block against excess resource consumption, etc.

7. Documents to be Written

Collectively, we decided on several documents that need to be written:

Taxonomy of Attacks

In order to defend a protocol against attacks, one must, of course, know the kinds of attacks that are possible. While the specifics differ from protocol to protocol, a number of general categories can be constructed.

Implementation Hints and Pitfalls Even if a protocol is sound, a host running it can be vulnerable if the protocol is implemented improperly. A variety of common errors can and do subvert the best designs.

Firewall Issues

Firewalls are both a common defense and a much-reviled wart on the Internet. Regardless, they are unlikely to go away any time soon. They have both strengths and weaknesses that must be considered when deploying them. Furthermore, some protocols have characteristics that are unnecessarily firewall-hostile; such practices should be avoided.

Workshop Report This document.

\section{Working Group Charters}

The actual text in the working group charter is likely to be something fairly simple, like

Protocols developed by this working group will be analyzed for potential sources of security breach. Identified threats will be removed from the protocol if possible, and documented and guarded against in other cases.

The actual charter text represents a policy enjoined and enforced by the IESG, and may change from time to time and from charter to 
charter. However, it essentially references and asks for text in documents conforming to the following, which may be very appropriate to include in the RFC.

9. Guidelines on writing Security Considerations in an RFC

A "threat" is, by definition, a vulnerability available to a motivated and capable adversary. CERT advisories are quite predictable given a knowledge of the target of the threat; they therefore represent an existence proof, but not a threat analysis. The point is to determine what attacks are possible ("capabilities" of a potential attacker) and formulate a defense against the attacks, or convincingly argue that the attack is not realistic in some environment and restrict use of the protocol to that environment.

Recommended guidelines:

All RFCs - MUST meaningfully address security in the protocol or procedure it specifies. It MUST consider that it is giving its data to "the enemy" and asking it to be delivered to its friends and used in the manner it intended. Consideration MUST be given to the ramifications of the inherent danger of the situation.

- MUST do "due diligence" to list the threats to which the protocol is vulnerable. Use of legal term does not imply legal liability, but rather the level of responsibility expected to be applied to the analysis. This discussion might occur throughout the document or in the security Considerations section; if it occurs throughout, it MUST be summarized and referenced in the Security Considerations section.

- MUST discuss which of those threats are * Ameliorated by protocol mechanisms (example: SYN attack is ameliorated by clever code that drops sessions randomly when under SYN attack)

* Ameliorated by reliance on external mechanisms (example: TCP data confidentiality provided by IPSEC ESP)

* Irrelevant ("In most cases, MIBs are not themselves security risks; If SNMP Security is operating as intended, the use of a MIB to change the configuration of a system is a tool, not a threat. For a threat analysis of SNMP Security, see RFC ZZZZ.")

* Not addressed by the protocol; results in applicability statement. ("This protocol should not be used in an environment subject to this attack") 
10. Core Security Mechanisms

A variety of security mechanisms exist today. Not all are welldesigned; not all are suitable for all purposes. The members of the workshop designated a number of protocols as "core". Such protocols should be used preferentially, if one of them has properties that match the needs of your protocol. The following were designated as core:

IPsec [RFC 1825] is the basic host-to-host security mechanism. It is appropriate for use any time address-based protection would have been used, including with such programs as rsh and rlogin. If and when platforms support user-based keying, this scope may be expanded.

One particular technique used by IPsec, HMAC [RFC 2104], is more generally useful. If cryptographic authentication but not secrecy is needed, and IPsec is not applicable, HMAC should be used.

ISAKMP/Oakley [ISAKMP drafts] is the basic key negotiation protocol for IPsec. As such, it should be deployed when IPsec is used. With the appropriate "domain of interpretation" document, it should be used to negotiate pairwise keys for other protocols.

DNSsec [RFC 2065] is not only crucial for protecting the DNS -cache contamination is the easiest way to launch active attacks -- it's also needed in many situations when IPsec is used.

Security/Multipart [RFC 1847] is the preferred way to add secured sections to MIME-encapsulated email.

Signed keys in the DNS. There is, as noted, widespread agreement that DNS records themselves must be protected. There was less agreement that the key records should be signed themselves, making them in effect certificates. Still, this is one promising avenue for Internet certificates.

X.509v3 is the other obvious choice for a certificate infrastructure. Again, though, there was no strong consensus on this point.

TLS [TLS draft] was seen by some as the preferred choice for transport-layer security, though there was no consensus on this point. TLS is less intrusive to the operating system than IPsec; additionally, it is easier to provide fine-grained protection this way. 
Some protocols were designated as "useful but not core". These were insufficiently general, too new, or were substantially duplicative of core protocols. These include AFT/SOCKS, RADIUS, firewalls, GSS-API, PGP, Kerberos, PGP-MIME, PKIX-3, the various forms of per-hop authentication (OSPF, RSVP, RIPV2), *POP, OTP, S/MIME, SSH, PFKey, IPsec API, SASL, and CRAM/CHAP. Obviously, entries on this list may move in either direction.

A few protocols were considered "not useful". Primarily, these are ones that have failed to catch on, even though they've been available for some time. These include PEM [RFC 1421, 1422, 1423, 1424] and MOSS [RFC 1848]. (The phrase "not useful" does not imply that they are incorrect, or are lacking in important information. However, they do not describe protocols that we are currently encouraging the use of.)

One security mechanism was deemed to be unacceptable: plaintext passwords. That is, no protocol that relies on passwords sent over unencrypted channels is acceptable.

\section{Missing Pieces}

Participants in the workshop identified three significant missing pieces: object security, secure email, and route security.

object security refers to protection for individual data objects, independent of transport. We have one such already -- Secure DNS -but we need a me general scheme. MIME is a candidate object framework, in part because it is the core of the two most widely used and deployed applications: the web and email. However, securing email has been problematic and the web is not that far in front.

Secure email is a critical need and has been for some time. Two attempts to standardize secure email protocols (PEM and MOSS) have failed to be accepted by the community, while a third protocol (PGP) has become a de facto standard around the world. A fourth protocol, an industry standard (S/MIME), has been gaining popularity. Both of these latter of entered the Internet standards process.

Route security has recently become a critical need. The sophistication of the attackers is on the rise and the availability of attacking toolkits has increased the number of sophisticated attacks. This task is especially complex because the requirement for maximum performance conflicts with the goal of adding security, which usurps resources that would otherwise enhance the performance of the router. 


\section{Security Considerations}

Security is not and cannot be a cookie cutter process. There is no magic pixie dust that can be sprinkled over a protocol to make it secure. Each protocol must be analyzed individually to determine what vulnerabilities exist, what risks they may lead to, what palliative measures can be taken, and what the residual risks are.

\section{Acknowledgments}

This RFC is largely based on the minutes compiled by Thomas Narten, whose work in turn was partly based on notes by Erik Huizer, John Richardson, and Bob Blakley.

14. References

[RFC 1825] Atkinson, R., "Security Architecture for the Internet Protocol", RFC 1825, August 1995.

[RFC 2104] Krawcyzk, H., Bellare, M., and R. Canett, "HMAC: Keyed-Hashing for Message Authentication", RFC 2104, February 1997.

[ISAKMP drafts] Works in Progress.

[RFC 2065] Eastlake, D., and C. Kaufman, "Domain Name System Security Extensions", RFC 2065, January 1997.

[RFC 1847] Galvin, J., Murphy, S., Crocker, S., and N. Freed, "Security Multiparts for MIME: Multipart/Signed and Multipart/Encrypted", RFC 1847, October 1995.

[TLS draft] Dierks, T., and C. Allen, "The TLS Protocol Version 1.0", Work in Progress.

[RFC 1421] Linn, J., "Privacy Enhancement for Internet Electronic Mail: Part I: Message Encryption and Authentication Procedures", RFC 1421, February 1993.

[RFC 1422] Kent, S., "Privacy Enhancement for Internet Electronic Mail: Part II: Certificate-Based Key Management", RFC 1422, February 1993 .

[RFC 1423] Balenson, D., "Privacy Enhancement for Internet Electronic Mail: Part III: Algorithms, Modes, and Identifiers", RFC 1423, February 1993. 
[RFC 1424] Kaliski, B. "Privacy Enhancement for Internet Electronic Mail: Part IV: Key Certification and Related Services", RFC 1424, February 1993.

[RFC 1848] Crocker, S., Freed, N., Galvin, J. and S. Murphy, "MIME object Security Services", RFC 1848, October 1995.

Appendix A. Attendees

$\begin{array}{ll}\text { Ran Atkinson } & \text { rja@inet.org } \\ \text { Fred Baker } & \text { fred@cisco.com } \\ \text { Steve Bellovin } & \text { bellovin@acm.org } \\ \text { Bob Blakley } & \text { blakley@vnet.ibm.com } \\ \text { Matt Blaze } & \text { mab@research.att.com } \\ \text { Brian Carpenter } & \text { brian@hursley.ibm.com } \\ \text { Jim Ellis } & \text { jte@cert.org } \\ \text { James Galvin } & \text { galvin@commerce.net } \\ \text { Tim Howes } & \text { howes@netscape.com } \\ \text { Erik Huizer } & \text { Erik.Huizer@sec.nl } \\ \text { Charlie Kaufman } & \text { charlie_kaufman@iris.com } \\ \text { Steve Kent } & \text { kent@bbn.com } \\ \text { Paul Krumviede } & \text { paul@mci.net } \\ \text { Marcus Leech } & \text { mleech@nortel.ca } \\ \text { Perry Metzger } & \text { perry@iermont.com } \\ \text { Keith Moore } & \text { moore@cs.utk.edu } \\ \text { Robert Moskowitz } & \text { rgm@htt-consult.com } \\ \text { John Myers } & \text { jgm@cMU.EDU } \\ \text { Thomas Narten } & \text { narten@raleigh.ibm.com } \\ \text { Radia Perlman } & \text { radia.perlman@sun.com } \\ \text { John Richardson } & \text { jwr@ibeam.jf.intel.com } \\ \text { Allyn Romanow } & \text { allyn@mci.net } \\ \text { Jeff Schiller } & \text { jis@mit.edu } \\ \text { Ted T'So } & \text { tytso@mit.edu } \\ & \end{array}$

Appendix B. Author Information

Steven M. Bellovin

AT\&T Labs Research

180 Park Avenue

Florham Park, NJ 07932

USA

Phone: (973) 360-8656

EMail: bellovindacm.org 
Full Copyright statement

Copyright (C) The Internet Society (1998). All Rights Reserved.

This document and translations of it may be copied and furnished to others, and derivative works that comment on or otherwise explain it or assist in its implementation may be prepared, copied, published and distributed, in whole or in part, without restriction of any kind, provided that the above copyright notice and this paragraph are included on all such copies and derivative works. However, this document itself may not be modified in any way, such as by removing the copyright notice or references to the Internet society or other Internet organizations, except as needed for the purpose of developing Internet standards in which case the procedures for copyrights defined in the Internet Standards process must be followed, or as required to translate it into languages other than English.

The limited permissions granted above are perpetual and will not be revoked by the Internet society or its successors or assigns.

This document and the information contained herein is provided on an "AS IS" basis and THE INTERNET SOCIETY AND THE INTERNET ENGINEERING TASK FORCE DISCLAIMS ALL WARRANTIES, EXPRESS OR IMPLIED, INCLUDING BUT NOT LIMITED TO ANY WARRANTY THAT THE USE OF THE INFORMATION HEREIN WILL NOT INFRINGE ANY RIGHTS OR ANY IMPLIED WARRANTIES OF MERCHANTABILITY OR FITNESS FOR A PARTICULAR PURPOSE. 